\title{
ATMOSPHERIC PRESSURE PLASMA GENERATION SYSTEM BASED ON PULSED VOLUME DISCHARGE FOR THE BIOLOGICAL DECONTAMINATION OF A SURFACE.
}

\author{
Ponomarev A. V. ${ }^{a, *}$, Spyrina A. V. ${ }^{a}$, Mamontov Y. I. ${ }^{a}$, \\ VOLKhina V. N. ${ }^{b}$, ZAKirov T. V. ${ }^{b}$, IOShChenko E. S. ${ }^{b}$ \\ ${ }^{a}$ Ural Branch of the Russian Academy of Sciences, Institute of Electrophysics, Amundsen Str. 106, Ekaterinburg, \\ Russia \\ ${ }^{b}$ Ural State Medical University, Repina str. 3, Ekaterinburg, Russia \\ * apon@iep.uran.ru
}

\begin{abstract}
The research introduces a system for pulsed volume discharges ignition at atmospheric pressure within gaps reaching $125 \mathrm{~mm}$. The corona discharge is used for the volume discharge initiation. A damping oscillations pulse generator is used as a high-voltage power supply. The pulse repetition rate reaches $1 \mathrm{kHz}$, while the rate of damping high-frequency harmonic oscillations can reach megahertz units. The volume discharge electric and spectral characteristics were analyzed. The study revealed that $\mathrm{O}_{2}^{+}$emission spectrum dominates in the UV region. The potential of using pulsed volume discharge for cleaning biological surfaces was demonstrated in the research. The survival rate for E. coli under the influence of 15 seconds long pulsed volume discharge has decreased by 30 times.
\end{abstract}

Keywords: pulsed volume discharge, corona discharge, damping oscillations pulse generator, optical emission spectrum, bio-decontamination.

\section{Introduction}

Low-temperature plasma has an extremely wide range of application. The main applications of lowtemperature plasma are, for example: plasma-assisted chemical vapour deposition, etching, polymerization, gas-phase synthesis, protective coating deposition, toxic and harmful gas decomposition, destruction of warfare agents, electromagnetic wave shielding, gas laser excitation and odour control, etc [1]. Successful use of low-temperature plasma for medical purposes has led to the appearance of a whole area of its practical use - plasma medicine [2 4].

There are many methods to generate atmosphericpressure low-temperature plasma. All of the methods can be categorized into 4 main groups: DBD - dielectric barrier discharges, corona discharges, $\mathrm{RF}$ and microwave discharges, and volume discharges. The most commonly applied method in experiments is the DBD method due to its structural simplicity and ease of implementation. Due to its low voltage level (tens of kilovolts) it allows the use of standard power supply sources. Its short-range action is the only disadvantage of the discharge method.

For instance in the researches on treatment of uneven surfaces [5] it is shown that the maximum possible height of the unevenness in the experiments does not exceed $2 \mathrm{~mm}$. This discharge type exists mainly in filamentary mode [6], but it has configurations for ignition of homogeneous discharges [7].

Corona discharge can exist in different modifications, be it an incomplete single-electrode or a complete double-electrode. It can run on direct or pulsed voltage and possess different voltage and power levels ranging from units of kilovolts and units of milli-watts [8] to hundreds of kilovolts and tens of kilowatts [9]. The advantage of this discharge type compared to the barrier discharge is its long-range action and ability to generate significantly higher volumes of lowtemperature plasma.

RF and microwave discharges are mainly used to produce plasma-jet and micro-plasma systems [10] and are widely used for medical purposes. The problem is such discharge ignition requires expensive inert plasma forming gases or their mixtures. Another inherent disadvantage of these discharges is their treatment area locality. The system for ignition of DBD RF discharge on Ar-air mixture is demonstrated in [11. Also when in use 12 the uniform RF discharge can burn in the air after it is ignited in the inert gases environment.

A pulsed volume discharge is of utmost interest for generating considerable amounts of low-temperature plasma in the range of tens of centimeters [13. These discharges do not use dielectric barrier layers, and as such are different from the recently widely researched atmospheric pressure glow discharges (APGD). The pulsed volume discharge and classical steady-state glow discharge are similar to one another, where the both methods have cathode and anode fall, faraday dark space and a positive column. At the same time the volume discharge significantly differs from the classical glow discharge in a number of ways. Firstly, the gas for the volume discharge needs to be pre-ionized, which can be accomplished with an auxiliary discharge 
such as a DBD, corona or spark discharge. Secondly, the volume discharge is a non-steady type of discharge. If the discharge duration is not artificially limited, it is bound to constrict into a single conducting channel. Another difference lies in its peak power density that can exceed $1 \mathrm{MW} / \mathrm{cm}^{3}$ significantly outweighing similar characteristics of the classical glow discharge.

It would be more appropriate to call this discharge a high-pressure glow discharge, but the term "volume discharge" became widely used before their similarities were established. The adjective "volume" emphasizes the fact that the characteristics of this discharge are determined primarily by the process in the discharge itself and are not related to its interaction with the chamber walls as in the classical glow discharge.

The volume discharge has wide practical use. It is this type of discharge that is used for laser pumping for various technological applications. Furthermore, it is used to construct plasma-chemical reactors and also to initiate and support various processes on the surface of solid bodies [14.

This research introduces a system for lowtemperature plasma generation through the use of pulsed volume discharge triggered by corona discharge at atmospheric pressure. The paper lists characteristics of the discharge and for the first time demonstrates the possibility to is it for surface biological decontamination.

\section{Experimental setup and methods}

To study the parameters of pulsed volume discharge and its use for biological decontamination of surfaces an equipment system consisting of DOPG-0.7 highvoltage generator (a detailed description is given in 15]) was used in the research.

The generator is capable of forming damping harmonic oscillations pulses with a frequency of up to $1 \mathrm{kHz}$. But the rate of high-frequency oscillations themselves can reach megahertz units. The maximum energy of the output pulse at optimal resistive load at frequency of $1 \mathrm{MHz}$ reached $0.4 \mathrm{~J}$. The advantage of such generator is its capability to repeatedly generate plasma between the discharge gaps. This results in the increase of plasma interaction time with the treated object in full possession of pulse mode advantages. It also increases the actual repetition rate of discharge pulses that will be equal to the multiplication of generator operation rate by the number of plasma generation moments per one pulse.

The discharge set to "multi-needle high-voltage electrode to grounded plate" was ignited between the gaps of $75 \mathrm{~mm}$ to $125 \mathrm{~mm}$. The electric measurement facilities included a P6015A high-voltage probe and current shunts. The shunts had a proper signal rise time of no more than 1 ns. A TDS 684B oscilloscope with a $1 \mathrm{GHz}$ bandwidth and Barth Electronics wide band signal attenuators were used for recording output pulses. Photo images were made with the Pentax

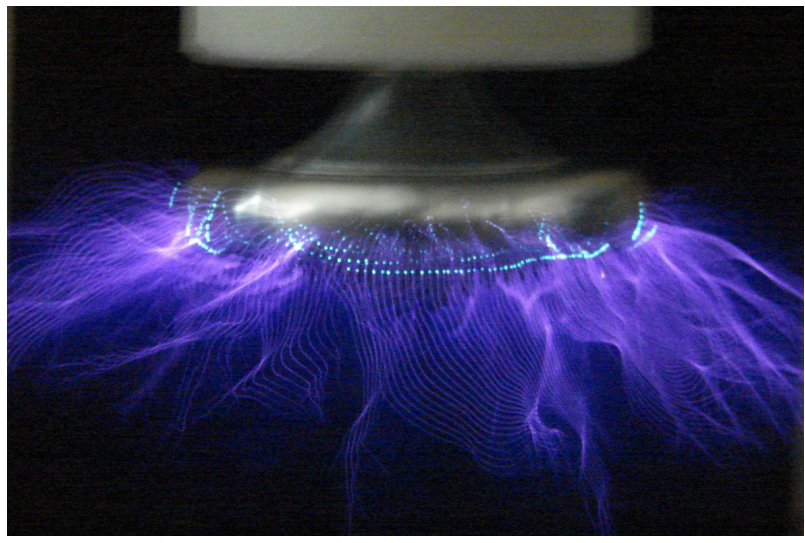

Figure 1. Incomplete single-electrode streamer corona discharge.

K-X camera. Optical characteristics were studied using a photomultiplier with UV-VIS spectrum region sensitivity (220-600 nm). Spectral measurements of the discharge were conducted using a device manufactured by "MORS" LLC (Russia, Troitsk). Spectra were recorded using a photoreceiver containing a diffraction spectrograph and multi-channel optical recording system, representing a joint of an image converter tube and a linear charge-coupled device. Two photorecievers were used to record spectra in wavelength ranges of 200-400 nm and 400-900 nm. Error of wavelength measurement for the first range was $+/-0.2 \mathrm{~nm}$, for the second - +/ $-0.5 \mathrm{~nm}$.

As an object of exposure the bacteria E.coli strain M17 were used during the operation. The nutrient medium in the Petri dish was a beef-extract agar.

\section{Experimental results}

\subsection{Electrical properties of pulsed volume discharge}

In the process atmospheric pressure gas discharge was ignited in two different modes. The first mode was the incomplete single-electrode corona discharge that exists in the area of high-voltage electrode. It ignites when there are large inter-electrode distances and field strength is weak enough so as not to perforate the gap. This discharge exists mainly in the streamer mode (Figure 1).

A photo depicting generator operating in the frequency mode was made with the $1 \mathrm{~s}$ long exposure and it is possible to see thin streamers, originating from bright spots at the external surface of the toroidal electrode made of aluminum. Supposedly these spots are the emission centers providing there is negative polarity at the high-voltage electrode. At such a polarity the high-voltage electrode is considered to be a cathode emitting electrons under the influence of the photoemission, autoemission and ion bombardment. In 13 it is noted that in some cases the aluminum can possess an abnormally high photo- and ion-electron emission. It is explained by the presence of films and 

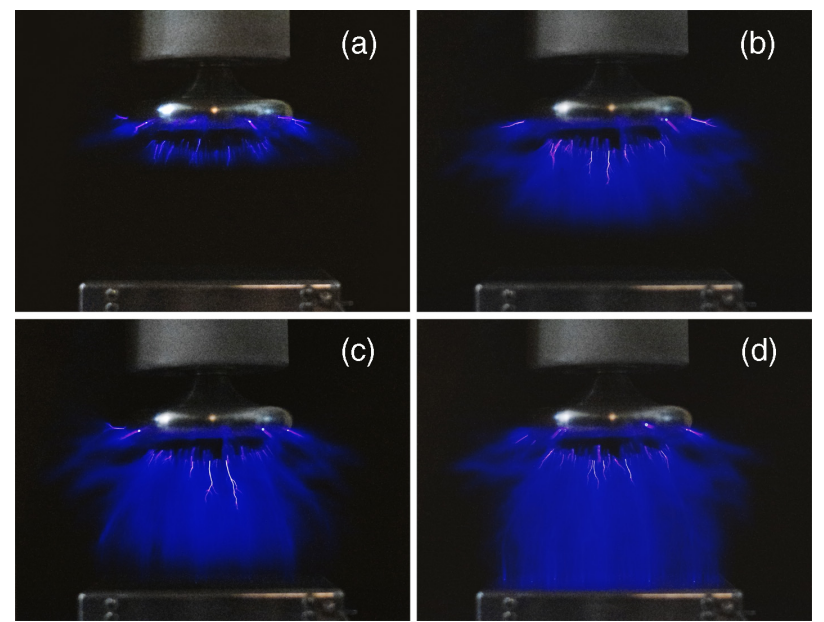

Figure 2. Development of the corona discharge from the side of high-voltage electrode $(a-c)$; emergence of pulsed volume discharge (d).
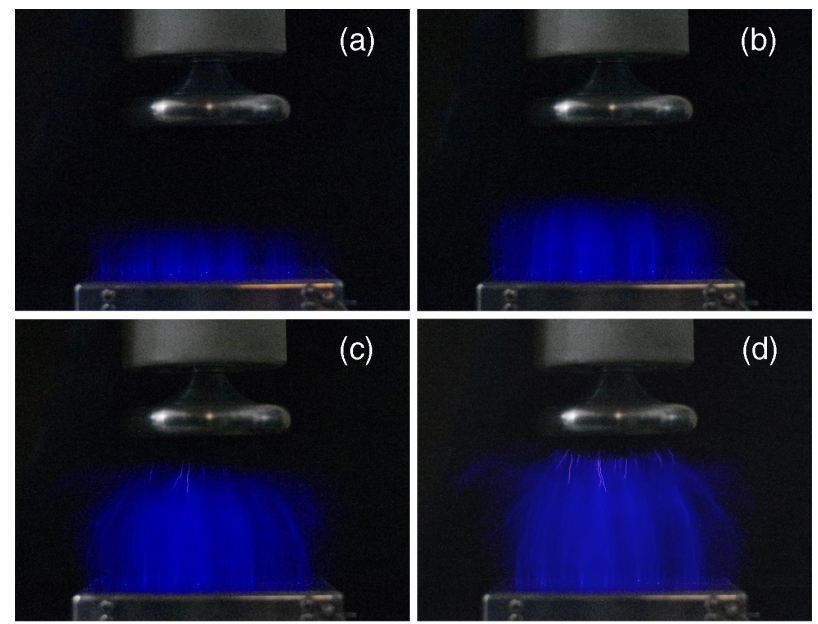

Figure 3. Development of a back corona discharge from the low-voltage electrode side $(a-d)$.

micro-particles on its surface. Thus several local emitting centers can create current of single to tens of amperes. The size of the spots is around $1 \mathrm{~mm}$ and less which exceeds the size of cathode spots in arc mode at indicated rate of current by 2 times. With each pulse the spots appear in a new place with some almost constant offset relative to the previous location. This leads to the appearance of bright intermittent stripes on the surface of the high-voltage electrode on the photo.

When reducing the inter-electrode distance the discharge proceeds to the second mode. Field strength in the inter-electrode gap becomes sufficient to ignite the pulsed volume discharge. Here corona discharge provides the necessary initial concentration of electrons for its ignition. Corona discharge also has the UV radiation facilitating the emergence of such a discharge type.

Figure 2 shows the photos of the volume discharge development from the high-voltage electrode side per one pulse.
The photos depict the moment of a single-electrode corona discharge emergence along with the development of incomplete streamers (Figure 2-a). Then the discharge develops towards the low-voltage electrode (Figure 2-b,c). Figure 2-d demonstrates that the gap is completely filled with discharge plasma that leads to the emergence of a volume discharge. The presence of a current in the low-voltage electrode circuit testifies to the emergence of such a discharge. In earlier research we supposed that we were dealing with the complete double-electrode corona discharge of the flash type. In [16] it was noted that positive charging of the corona electrode could lead to the formation of large current impulses with a magnitude significantly higher than the steady-state positive corona current. Such a regime, they called it a flashing corona discharge, and these current impulses are associated with the formation and development of positive streamers. Although in our case, judging by the photos of the discharge development, it is not the streamer mode, but diffusive mode that the discharge develops in. This allows us to label it the pulsed volume discharge.

Since the output voltage of a generator is a variable, the pulsed volume discharge initiation can occur either from the high-voltage electrode side, in the case of a negative corona, or from the grounded electrode in back corona [17] mode.

Figure 3 shows the photos of discharge development stages from the low-voltage electrode side. There is a possibility for the back corona to emerge on oxide or organic films, or on the inclusions into the electrode material. The plasma front starts its movement towards the high-voltage electrode and the gap is completely filled with plasma. In this case, the low-voltage electrode functions as a cathode.

As opposed to the high-voltage electrode, the surface of the low-voltage electrode does not have sharp points, thus amplifying the field strength locally. Therefore, practically no streamers are formed from it unlike with the corona development from the highvoltage electrode side. The source of electrons is the electrode's surface, exposed to discharge photons and ions. Ions accelerate in a length insignificant to cathode fall, the length of which [18] is estimated at 100 $\mu \mathrm{m}$ in such conditions. But it should be noted that in the area of high currents, reaching $10 \mathrm{~A}$ in pulse, the discharge near the surface of the low-voltage electrode is heterogeneous. Along with the homogeneous illumination brighter local spots appear on the surface that also has a size of about $1 \mathrm{~mm}$ and less. Upon close examination of the discharge photos it was noted that given spots are the source of plasma torchers, possessing higher plasma density than its remaining volume. Making a comparison to the abnormal glow discharge will explain the emergence of local areas of cathode surface with more intensive emission. By increasing the current in the glow discharge the area of cathode surface increases [19. By further increasing the current and it is no longer possible to increase 

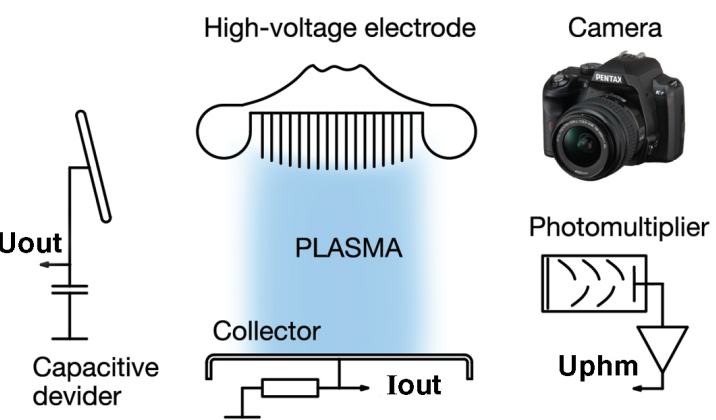

Figure 4. Schematic of the experimental setup.

the emission surface area, the glow discharge proceeds to the abnormal stage. At this the emission current density increases and the discharge current continues to grow.

This way at discharge currents reaching 10 A during the experiment, homogeneous photo- and ion-electron emission is not enough, which leads to the emergence of local areas with increased emission.

In 20] it is indicated that anode and cathode spots in homogeneous glow discharges of homogeneous gas discharges have a non-arc nature. Being the center of ionization, they provide the ion and electron flow to maintain the gas discharge. When reducing the amplitude of the current the number of spots gradually decreases up to their full disappearance. In this case, the discharge illumination near the electrodes becomes homogeneous.

A schematic of the experimental setup is shown on Figure 4

Waveforms of the output voltage (a), the signal from the photomultiplier (b) and the collector current (c) per a single pulse at the oscillation frequency of $680 \mathrm{kHz}$ are shown on Figure 5. The voltage of the high-voltage electrode was measured with a capacitance divider. The light emission from the discharges was detected by a photomultiplier. The collector current (flat electrode at ground potential) was measured with a resistive shunt. Unfortunately, the authors have to acknowledge the low accuracy of the measurement of the amplitude of the first $\mathrm{RF}$ oscillation periods since the plasma-filled high-voltage gap distorts the readings of the capacitance divider.

The waveform of a collector current indicates that the volume discharge in a gap exists only during the first high-voltage half-cycles. Subsequent pulses of lower amplitude are not capable of igniting the volume discharge due to insufficient field strength in a gap. But the light emission continues to be present in the absence of collector current signal.

It is evidence of the fact that at lower voltages the discharge proceeds to the stage of incomplete corona discharge in the area of the high-voltage electrode.

The current asymmetry of a collector is a result of the applied asymmetric form of the electrode configu-

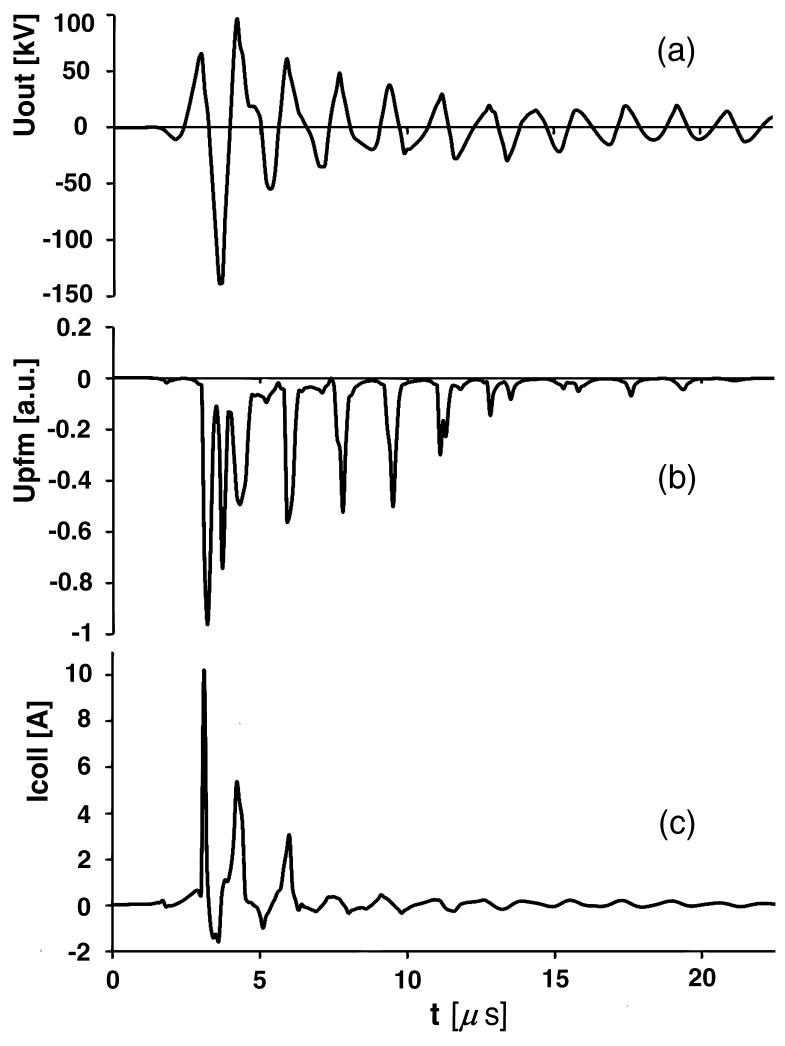

Figure 5. Waveforms of generator operation: output voltage (a), photomultiplier output signal (b), grounded plate (collector) current (c).

ration: multi-needle high-voltage electrode-grounded plate. Positive polarity current predominates, where a flat-grounded collector acts as a cathode. In this case only the volume discharge ignites effectively. It is probably connected with the fact that when a flat polished low-voltage electrode acts as a cathode, the voltage at the gap is higher and the field strength is enough to ignite the volume discharge. When using a symmetrical configuration of electrodes the current of volume discharge also becomes symmetrical.

\subsection{Emission spectrum of the pulsed volume discharge}

The optical emission spectrum (OES) allows the analysis of the radiation emitted by the atoms, ions, molecules and radicals. In [21] in the emission spectrum of the air surface discharge plasma the emission of $\mathrm{N}_{2}$ lines dominate in the wavelength range of 250-700 nm. Lines $\mathrm{O}$ and $\mathrm{OH}$ can also be observed. According to the authors it is reactive $\mathrm{O}$ and $\mathrm{OH}$ that play a significant role in the activation.

The authors [22] detected the emission spectra of DC discharges in the ultraviolet, visible and near infrared regions. They observed the spectrum of the second positive nitrogen system in the UV region, which was accompanied by the first positive system in the visible region. This points to the production of $\mathrm{A}^{3} \sum_{u}^{+}$states of $\mathrm{N}_{2}$, the long-living meta-stable states that are important as the energy reservoir, supporting 

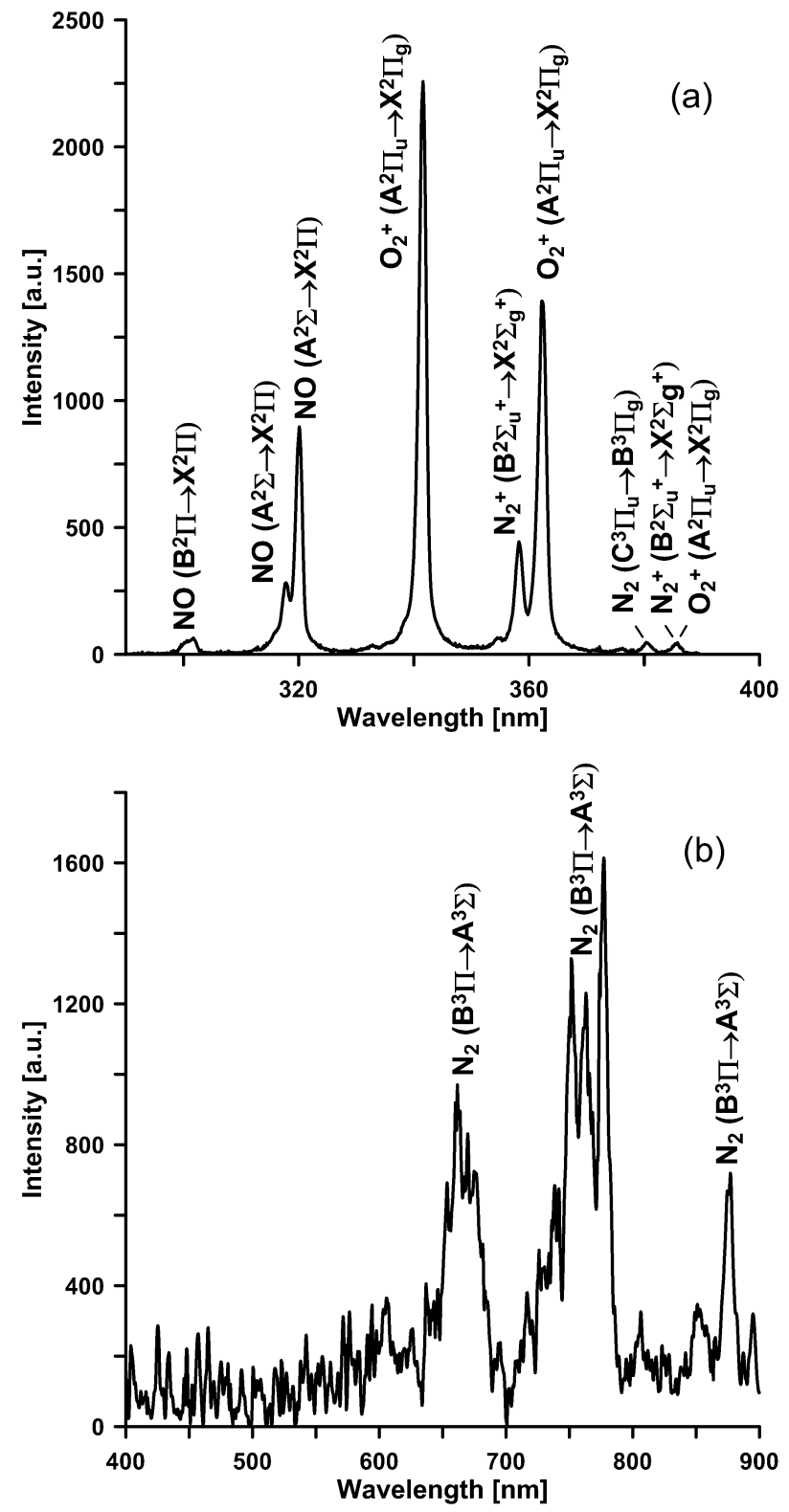

Figure 6. Emission spectrum of the pulsed volume discharge: (a) - wavelength range of 200-400 $\mathrm{nm},(\mathrm{b})$ - 400-900 $\mathrm{nm}$.

chemical reactions running in plasma. Besides that, spectra show the emission of $\mathrm{OH}$ and $\mathrm{NO}$ radicals, the emission of the first negative system of $\mathrm{O}_{2}^{+}$and atomic lines N, O, H. Due to their high reactivity and bactericidal ability these emissions play a key role during the biochemical disinfection, surface and water sterilization. In the 23 research, when studying the optical emission of the air plasma in the wavelength range of 250-800 nm, the dominant lines of $\mathrm{N}_{2}$ emission as well as spectrum and O-atom emission were also detected. It is known that $\mathrm{O}$ atoms are very active and play a very important role in the applications of plasma in medicine. In [24] the authors studied the spectral range of $180-900 \mathrm{~nm}$ emission of the dielectric barrier discharge of atmospheric cold plasma. Strong lines of $\mathrm{N}_{2}$ and $\mathrm{N}_{2}^{+}$emissions were detected, but there

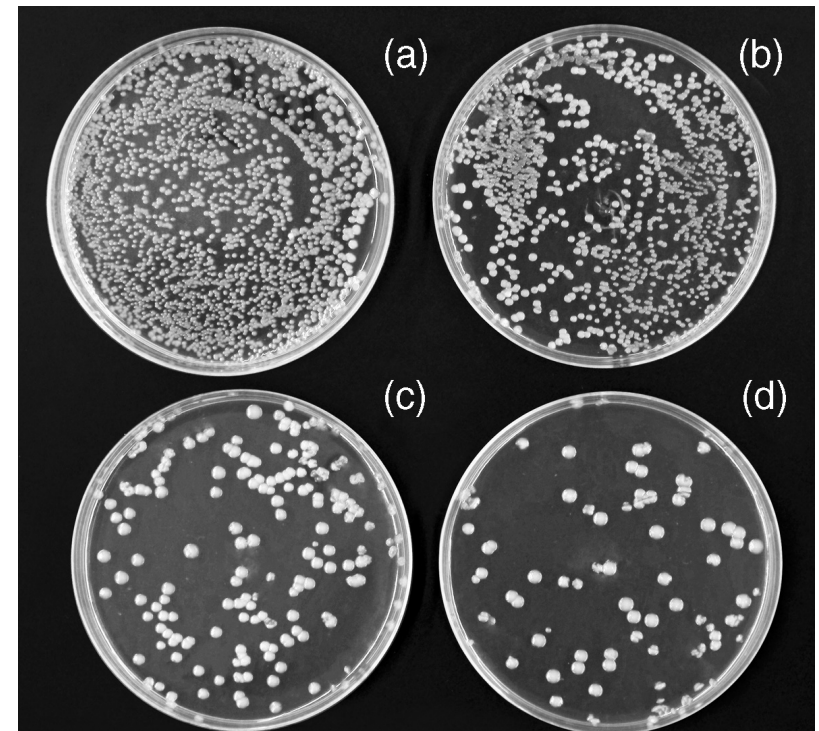

Figure 7. Petri dishes with contaminated agar: untreated control (a); treated by pulsed volume discharge for $5,10,15$ s respectively $(b-d)$.

were not detected any main peaks corresponding to active oxygen.

Figure 6 demonstrates the emission spectra of pulsed volume discharge within ranges of 200-400 nm (a) and 400-900 nm (b). As seen on the Figure 6 (a), emission lines of $\mathrm{O}_{2}^{+}, \mathrm{NO}, \mathrm{N}_{2}$ and $\mathrm{N}_{2}^{+}$molecules are present in the ultraviolet spectrum region, whereas the visible region is presented by the emission spectrum of the first positive nitrogen system (Figure 6(b)).

Thus our case observes not quite a typical spectrum of air plasma that was noted by the authors in researches 21 24 within the ultraviolet region. Namely the second positive nitrogen system is virtually absent and the most present lines are the lines of $\mathrm{O}_{2}^{+}$ emission. There is no data on the bactericidal activity of the positive oxygen ion, but it is obvious that it is a strong oxidant with the activation energy of about $11 \mathrm{eV}$.

The positive aspect of the given technology is the presence of NO nitrogen oxide in the discharge. In many researches, including [3, 25], this compound is named as the main healing factor in plasma medicine.

\subsection{Bactericidal properties of pulsed volume discharge}

The research includes a study on the bactericide properties of the pulsed volume discharge by using it to treat the surface of contaminated agar in Petri dishes of $90 \mathrm{~mm}$ in diameter. In the experiment, Petri dishes were mounted on a grounded mobile carriage, with which the agar had electrical contact.

E-coli were selected as the object of exposure which is the most commonly used bacteria to evaluate the effectiveness of the decontamination process in analogous experiments. One of the exposure results is shown on Figure 7. In the upper left corner a photo 


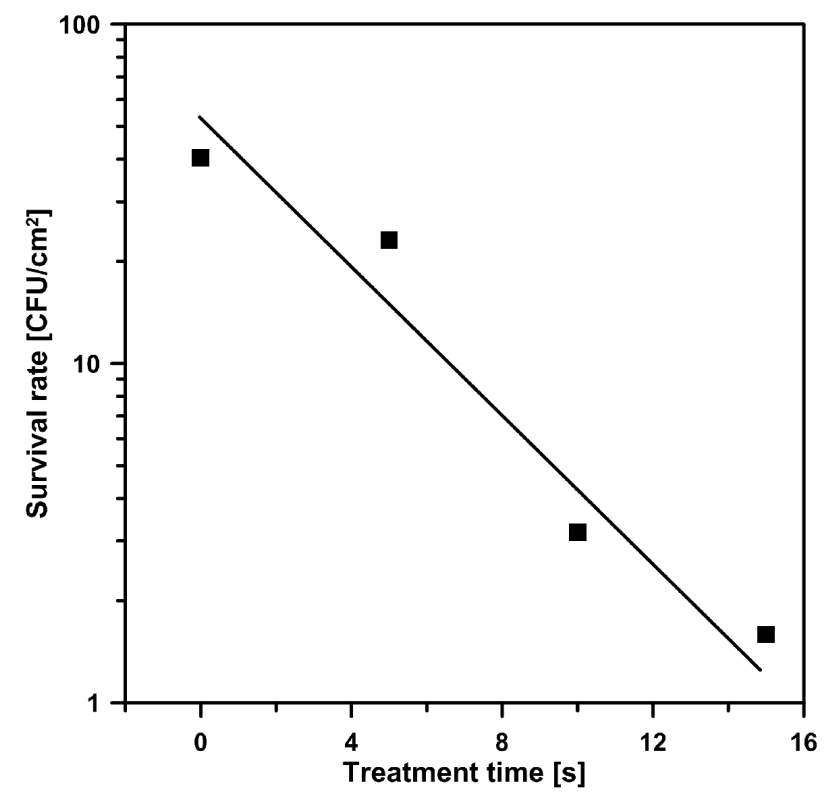

Figure 8. Survival curve of e-coli exposed to the pulsed volume discharge.

of the untreated control sample is shown. Three subsequent photos show the treatment results with exposures of 5, 10 and 15 s. On Figure 8 these results are shown as a dependency curve of CFU number per one $\mathrm{cm}^{2}$ of the treatment time.

Repetition rate of output pulses used in this experiment was $250 \mathrm{~Hz}$ at a frequency of RF oscillations which equalled to $0.7 \mathrm{MHz}$. The output power of the generator in this experiment was less than $100 \mathrm{~W}$. The distance between the rounded multi-needle highvoltage electrode with a diameter of $105 \mathrm{~mm}$ and the surface of the agar amounted to $100 \mathrm{~mm}$. After discharge exposure the dishes were placed in a $37^{\circ} \mathrm{C}$ incubator for about $18 \mathrm{~h}$.

\section{Discussion and conclusion}

The main question for any technology of biological decontamination using low temperature plasma is which of the plasma's exposure factors are destructive to living organisms? Is it the effect of the electric field, UV radiation, ROS and nitrogen compounds or is it the influence of charged particles? Perhaps it is just the burning effect of a plasma streamer or a filament with high power density.

Unfortunately our study cannot give an unambiguous answer to this question. Experiments have demonstrated that bacteria die only when the surface of contaminated agar represented the electrode of a discharge system. When a Petri dish was placed in the middle of the discharge gap under the floating potential, the decontamination effect was insignificant. Perhaps it is connected with the absence of atomic oxygen in the positive column of the discharge. Apparently the near-electrode processes play the main role in the decontamination procedure.
The significance of the near-electrode processes role is shown in [26], where it is said that cathode fall has the most interesting impact on the surface. For example, many short-living active species are not able to reach the treated surface from a discharge. Only some long-lived radicals such as ozone can reach the surface. Only active species produced in the cathode fall effectively reach the surface because the cathode fall is very close to the surface.

Spots-segments with increased emission (that were also detected on the agar surface during its treatment) probably play an important role in the decontamination process. Based on the light intensity they have significantly higher VIS-UV radiation intensity compared to remaining treated surface. In addition, the segments have high density of ions bombarding the surface. Also the local thermal effect on the bacteria in these spots cannot be excluded.

It may be noted that the examined discharge configuration of high-voltage gap of around $100 \mathrm{~mm}$ is not effective for decontamination of flat surfaces. The biggest part of pulse energy is spent in a discharge column and just a small part of it dissipated in the near-surface layer. In that respect DBD-based systems at inter-electrode gaps measuring in millimeters with the same power level in the discharge are more effective. But its disadvantage of short-range action has already been mentioned above. The benefits of our biological decontamination system can be displayed during the treatment of large non-flat surfaces.

The research paper demonstrates only the potential to use the pulsed volume discharge for biological decontamination of a surface. To address other issues in every individual case it is necessary to select the most effective discharge gap configuration and alight power supply parameters according to it.

Nevertheless, the authors believe that pulsed volume discharge has great potential for decontamination purposes since it is a diffuse discharge with high peak power density, which, as already has been mentioned, can exceed $1 \mathrm{MW} / \mathrm{cm}^{3}$. It will allow creating on its basis powerful gas discharge systems for biological decontamination of surfaces. Furthermore, the absence of streamer and filamentary modes in the discharge will allow uniformly treating objects with a large surface area.

\section{Acknowledgements}

The reported study was supported by Russian Foundation for Basic Research (RFBR), research project No. 15-0801707-a.

\section{References}

[1] A. P. Napartovich. Overview of atmospheric pressure discharges producing nonthermal plasma. Plasmas and Polymers, 6(1/2):1-14, 2001.

[2] M. Laroussi. Low-temperature plasmas for medicine? IEEE Trans. Plasma Sci., 37(6):714-725, 2009. 
[3] G. Fridman and Gutsol A. Shekhter A. B. Vasilets V. N. Fridman A. Friedman, G. Applied plasma medicine. Plasma Process. Polym., 5(6):503-533, 2008.

[4] M. G. Kong, Nosenko T Shimizu T Dijk J Kroesen G, Morïñ Ąll G, and Zimmermann J L. Plasma medicine: an introductory review. New J. Phys., 11(11):1-26, 2009.

[5] H. Ayan, Gutsol A Mukhin Y Starikovskii A Fridman A Staack D, Fridman G, and Friedman G. Application of nanosecond-pulsed dielectric barrier discharge for biomedical treatment of topographically non-uniform surfaces. J. Phys. D: Appl. Phys., 42(12):1-5, 2009.

[6] U. Kogelschatz. Dielectric-barrier discharges: Their history, discharge physics, and industrial applications. Plasma Chemistry and Plasma Processing, 23(1):1-46, 2003.

[7] H. Ayan, Vasilets V N Fridman A Fridman G, Gutsol A F, and Friedman G. Nanosecond-pulsed uniform dielectric-barrier discharge. IEEE Trans. on Plasma Sci., 36(2):504-508, 2008.

[8] D. Dorranian and Sari A. H. Nasr S. Amini L. Soudi M. R., Jamshidi M. M. Sterilization of esherichia coli and the microorganisms of turmeric samples with corona discharge plasma. J. of Theoretical and Appl. Phys., 4(3):28-36, 2010

[9] G. A. Mesyats, Rukin S. N. Slovikovsky B G Timoshenkov S P Ponomarev, A. V., and Bushlyakov A I. 1-mv, 500-hz all-solid-state nanosecond driver for streamer corona discharge technologies. High-Power Particle Beams, 2000 13th International Conference, pages 192-195, 2000.

[10] S. Uhm Han and Guang S. Cho Eun, H. Choi. Sterilization of microbes by using various plasma jets. $J$. of the Korean Phys. Society, 60(6):897-902, 2012.

[11] F. Sohbatzadeh and Mahdavi H. Omidi Z. Mirzanejhad, S. Characterization of argon/air atmospheric pressure capacitively coupled radio frequency dielectric barrier discharge regarding parasitic capacitor at $13.56 \mathrm{mhz}$. J. of Theoretical and Appl. Phys., 6(32):1-6, 2012.

[12] Li He-Ping and Guo Li Cheng-Yu Bao Wen-Ting Sun, Hua-Bo Wang. Electrical features of radio-frequency, atmospheric pressure, bare-metallic-electrode glow discharges. Plasma Chem. Plasma Process., 27(5):529-545, 2007.

[13] Yu. D. Korolev and G. A. Mesjac. Physics of Pulsed Breakdown in Gases. URO-PRESS, 1998.

[14] V. V. Osipov. Self-sustained volume discharge. Physics - Uspekhi, 43(3):221-241, 2000.

[15] A. V. Ponomarev and Pedos-M. S. Sergeev A. G.-Ustyuzhanin A. V. Podymova A. S. Gusev, A. I. High-frequency generator based on pulsed excitation of the oscillating circuit for biological decontamination. Pulsed Power Conference (PPC), 2013 19th IEEE, pages 1-5, 2013.

[16] I. V. Timoshkin and Wilson M. P. Given M. J. MacGregor-S. J. Wang Tao Anderson J. G. Maclean, M. Bactericidal effect of corona discharges in atmospheric air. IEEE Trans. on Plasma Sci., 40(10, part

1):2322-2333, 2012.
[17] T. Czapka and R. Kacprzyk. Non-thermal plasma reactor with back corona discharge electrode. J. of Phys.: Conference Series, 301(1):1-4, 2011.

[18] J. J. Shi and M. G. Kong. Cathode fall characteristics in a dc atmospheric pressure glow discharge. J. Appl. Phys., 94(9):5504-5513, 2003.

[19] A. F. Gutsol and W. R. Pyle. Negative corona 'tufts'. Plasma Sources Sci. Technol., 23(5):1-5, 2014.

[20] Yu. Akishev and Kochetov I. Napartovich A. Trushkin N. Karalnik, V. High-current cathode and anode spots in gas discharges at moderate and elevated pressures. Plasma Sources Sci. Technol., 23(5), 2014.

[21] D. Wang and Feng K. Zhang X. Liu D. Yang-S. Zhao, D. The cold and atmospheric-pressure air surface barrier discharge plasma for large-area sterilization applications. Appl. Phys. Lett., 98(16):1-3, 2011.

[22] J. Machala and Hensel K. Jedlovsky I. Lestinska L. Foltin-V. Martisovits V. Morvova M. Janda, M. Emission spectroscopy of atmospheric pressure plasmas for bio-medical and environmental applications. J. of Molecular Spectroscopy, 243(2):194-201, 2007.

[23] Wu ShuQun and ZiLan X. Yuan P. XinPei, L. A touchable pulsed air plasma plume driven by dc power supply. IEEE Trans. on Plasma Sci., 38(12):3404-3408, 2010.

[24] D. Ziuzina and Cullen P. J. Keener K. M. Bourke-P. Patil, S. Atmospheric cold plasma inactivation of escherichia coli in liquid media inside a sealed package. J. of Appl. Microbiology, 114(3):778-787, 2013.

[25] A. B. Shekhter and Rudenko T. G. Pekshev A. V.Vanin F. Serezhenkov, V. A. Beneficial effect of gaseous nitric oxide on the healing of skin wounds. Nitric oxide: biology and chemistry, 12(4):210-219, 2005.

[26] R. Ono and Oda T. Nakagawa, Y. Effect of pulse width on the production of radicals and excited species in a pulsed positive corona discharge. J. of Phys. D: Appl. Phys., 44(48):1-14, 2011. 